\title{
«Cyber-Docs» in der Apotheke?
}

Ärztliches Handeln hat eine neue Facette erhalten. In der Offizin soll neben Medikamenten und Kräutertees in speziell eingerichteten Séparées noch eine andere professionelle Dienstleistung bezogen werden können: Ein Basler Unternehmen, mehrheitlich im Besitz von Apothekern, mit dem Namen medicopharm bietet den «Cyber-Doktor» in der Apotheke an. Das ärztliche Beratungsgespräch kann damit künftig zwischen Patient, Apotheker und einem per Videosystem zugeschalteten Telemedizinarzt zu dritt geführt werden. Der Teledoc diagnostiziert und verschreibt aus der Telefonzentrale, der Apotheker verkauft und kassiert vor Ort, der Hausarzt wird obsolet und substituiert. 60 bis 90 Franken cash soll diese Form von Ferndiagnose und -therapie kosten dürfen.

Für die einen mag der Versuch einer vertikalen Integration von Ärztin oder Arzt in die Apotheke eine gewisse betriebswirtschaftliche Logik beinhalten, andere mögen darin eine Reaktion des Marktes auf alternative Formen in der Medikamentendistribution sehen, wie sie sich in den letzten Jahren etabliert haben.

Der Zentralvorstand der FMH hat von diesem neuen Angebot, das von einer Krankenversicherung und dem Generikahersteller Sandoz unterstützt wird, mit Staunen und Verwunderung Kenntnis genommen.

Der Zentralvorstand ist der Meinung, dass zwischen allgemeiner Informationsbeschaffung über gesundheitsrelevante Themen, für die heut- zutage mit Selbstverständlichkeit und zu Recht die verschiedensten elektronischen Medien eingesetzt werden, und einer an einem gesundheitlichen Problem orientierten ärztlichen Konsultation weiterhin grundsätzliche Unterschiede bestehen. Abgesehen von der haftungsrechtlichen Unsicherheit von bitvermittelter Diagnose und Therapie sieht sich der Zentralvorstand der FMH zu einer grundsätzlichen Stellungnahme zum Schutz des Arzt-Patienten-Verhältnisses veranlasst. Es zeugt von einem zweifelhaften Berufsverständnis, wenn die ärztliche Konsultation auf einen reinen Leistungsverkauf reduziert und der besondere zwischenmenschliche, relationale Charakter der Beziehung zwischen Arzt/Ärztin und Patient negiert wird. Dem Schutz und der Pflege dieses individuellen und humanen Aspektes der ärztlichen Tätigkeit hat unser besonderes Augenmerk zu gelten. Informationstechnologien sind Hilfsmittel zur Datenbeschaffung und Datenspeicherung. Sie sind nützliche Mittler zur Unterstützung des diagnostischen oder therapeutischen Prozesses, sie können aber nicht Teil des ärztlichen Handelns werden. So zweifeln wir auch nicht daran, dass die Protagonisten dieses Angebotes bald merken werden, was den Patienten schon von jeher bewusst ist, nämlich, dass ärztliche Leistungen anders konfektioniert sind als Hustensirup oder Antibiotika.

Ludwig T. Heuss, Mitglied des FMH-Zentralvorstands 


\section{«Cyber-Docs» en pharmacie?}

L'activité médicale vient de se trouver un nouveau créneau. L'officine qui servait à la vente de médicaments et de tisanes devrait désormais proposer une nouvelle prestation professionnelle dans un local séparé, spécialement aménagé pour l'occasion. En effet, une entreprise bâloise dénommée «medicopharm», principalement dans les mains de pharmaciens, offre les services d'un cyberdocteur exerçant la médecine en pharmacie. L'entretien médical peut avoir lieu entre patient, pharmacien et un système vidéo relié à un médecin par télécommunication de manière tripartite. Le «télémédecin» diagnostique et prescrit par ligne téléphonique interposée, le pharmacien vend le produit conseillé et encaisse sur place, se substituant au médecin de famille en le rendant obsolète. Ce type de diagnostic et de thérapie à distance devrait coûter entre 60 et 90 francs payés comptants.

Si pour d'aucuns, cette tentative d'intégration verticale du médecin dans la pharmacie contient une certaine logique d'économie d'entreprise, d'autres peuvent y voir une réaction du marché envers les formes alternatives de distribution de médicaments nouvellement établies ces dernières années.

Face à cette nouvelle forme d'offre, qui bénéficie du soutien d'une assurance-maladie et du producteur de génériques Sandoz, le Comité central de la FMH réagit avec étonnement et surprise.
Le Comité central est d'avis qu'entre des informations générales données sur des thèmes importants pour la santé, devenues aujourd'hui monnaie courante à travers les divers médias électroniques et une consultation médicale sur un problème de santé donné, d'énormes différences existent. Mis à part les doutes qu'induit ce type de diagnostic et de traitement sur le plan du droit en matière de responsabilité, le Comité central de la FMH tient à prendre fondamentalement position pour la protection de la relation médecin-patient. C'est faire en effet peu de cas de cette relation, de son aspect individuel et humain et de la profession médicale en général, que de réduire la consultation à un simple objet de vente de prestations. La protection et la pérennité de l'aspect individuel et humain de l'activité médicale sont dignes de tous nos égards. Les technologies d'information sont des moyens de créer des données et de conserver celles-ci. Elles servent à conforter le processus diagnostique ou thérapeutique, mais ne sauraient faire partie de l'acte médical. Nous ne doutons pas non plus que les protagonistes de cette idée reconnaîtront bientôt, ce le patient sait depuis toujours, que les prestations médicales fonctionnent différemment que la préparation d'un sirop pour la toux ou d'un antibiotique.

Ludwig T. Heuss, membre du Comité central 


\section{Zukunft Medizin Schweiz: Die Diskussion ist eröffnet}

* Der Bericht, der auch in französischer Sprache vorliegt, kann gratis bestellt werden beim Generalsekretariat der SAMW, Petersplatz 13, 4051 Basel, Tel. 0612699030 E-Mail: mail@samw.ch.
Die Schweizerische Akademie der Medizinischen Wissenschaften (SAMW), die Medizinischen Fakultäten und die FMH haben im Sommer 2002 das Projekt «Zukunft Medizin Schweiz» lanciert. Vorangegangen war eine breite öffentliche Diskussion; diese hatte gezeigt, dass die Medizin in der Schweiz einige Lücken aufweist und dass ein Ungleichgewicht besteht zwischen den postulierten Zielen und den erreichten Resultaten.

Die Projektverantwortlichen haben in der Folge eine interdisziplinäre Arbeitsgruppe («Expertengruppe») beauftragt, über die grundlegenden Werte der Medizin und notwendige Veränderungen nachzudenken und sich darüber zu verständigen, welches die Ziele, die Zuständigkeiten und die Grenzen der Medizin seien.
Ende 2004 liegt der Bericht dieser Gruppe nun vor; er trägt den Titel «Ziele und Aufgaben der Medizin zu Beginn des 21. Jahrhunderts». Die Projektverantwortlichen erachten diesen Bericht als wichtig, und sie möchten mit einer Sonderausgabe des SAMWbulletins, die der aktuellen Ausgabe der Schweizerischen Ärztezeitung beiliegt, alle Ärztinnen und Ärzte der Schweiz darüber informieren und zur Diskussion einladen*.

Unsere Kolleginnen und Kollegen, die medizinischen Fachgesellschaften und weitere interessierte Organisationen und Personen sind eingeladen, schriftlich oder anlässlich eines Forums am 16. Dezember 2004 zum Bericht Stellung zu nehmen. Wir sind gespannt auf Ihre Reaktionen.

Prof. Peter M. Suter, Genf, Präsident SAMW

\section{La médecine en Suisse demain: le débat est ouvert}

* Le rapport, qui existe également en allemand, peut être commandé gratuitement au secrétariat général de l'ASSM, Petersplatz 13, 4051 Bâle, tél. 06126990 30, e-mail: mail@samw.ch.
L'Académie suisse des sciences médicales (ASSM), les Facultés de médecine et la FMH ont lancé en été 2002 le projet «La médecine en Suisse demain»; il fait suite à un vaste débat public qui a mis en lumière quelques lacunes de la médecine suisse et surtout qui a fait ressortir un certain déséquilibre entre les objectifs souhaités et les résultats obtenus.

Depuis lors, un groupe de travail pluridisciplinaire («groupe d'experts») a été mandaté par les responsables du projet pour mener une réflexion par rapport aux valeurs essentielles de la médecine, aux remodelages à envisager ainsi que pour tenter de trouver un consensus au sujet des buts, des compétences et des limites de la médecine.
En cette fin d'année 2004, le rapport de ce groupe est disponible. L'importance de ce texte intitulé «Buts et missions de la médecine au début du 21 $21^{\mathrm{e}}$ siècle» justifie à nos yeux une édition spéciale du bulletin ASSM, annexé au numéro actuel du Bulletin des médecins suisses, dont le but est d'informer tous les médecins suisses et de lancer une discussion*.

Nos collègues, les sociétés de spécialistes ainsi que d'autres organisations et personnes intéressées sont invités à se prononcer par écrit sur ce rapport ou à l'occasion d'un forum qui se tiendra le 16 décembre 2004. Nous restons très intéressés par vos diverses réactions!

Prof. Peter M. Suter, Genève, Président ASSM 